\title{
ANATOMICALLY-BASED 3D FACE AND ORAL CAVITY MODEL FOR CREATING VIRTUAL MEDICAL PATIENTS
}

\author{
G. Moschos, N. Nikolaidis, I. Pitas \\ Department of Informatics \\ Artificial Intelligence and Information Analysis laboratory \\ Aristotle University of Thessaloniki \\ 54124 Thessaloniki, Greece \\ Tel,Fax: +30-231-099-6304 \\ e-mail: pitas@zeus.csd.auth.gr
}

\begin{abstract}
This paper presents a new hierarchical, modular and scalable model of a human face and oral cavity based on anatomical data taken from the Visible Human Project (National Institute of Health). The described model can further be adapted to any particular face and oral cavity of a human head by means of a Finite Elements Method (FEM). The final aim will be to construct functional virtual medical patients.
\end{abstract}

\section{INTRODUCTION}

Creating face and oral cavity functional models is very important for a number of applications in medicine, dentistry and speech pathology. Modelling techniques can be classified in automatic / semiautomatic and manual ones. Manual modelling techniques are the most labor-intensive. However, due to the geometrical complexity of the internal head structures and conformations, automatic/semiautomatic modelling techniques often yield poor results and thus one has to resort to manual modelling.

The first attempt for modelling a human face was made by F. I. Parke[1]. His pioneering work has been followed by others until 1987 when the first, publicly available, simplistic but generic face model namely the CANDIDE model, appeared, thanks to M. Rydfalk [2]. Its current version (CANDIDE-3) made by J. Ahlberg [3] incorporates new features and nodes to make the model more realistic and compliant with the emerging MPEG-4 standard.

To date, little attention has been paid on modelling and animating inner head structures, such as the intraoral cavity, despite its profound impact on intra-human communication and medical/dental research. O. Engwall [4] has applied semi-automatic methods on MRI, EPG and Electromagnetic Articulography (EMA) data in order to obtain intraoral models for speech generation simulation. Maureen
Stone et al. [5] used tagged Cine-Magnetic Resonance Imaging (tMRI) data for tongue modelling while Y. Laprie et al. [6] utilized X-rays for the same purpose.

However, none of these methods have produced a combined model of face, lips, tongue, teeth, palate and oral cavity. We have created such a 3D surface model containing 754 nodes (70 for facial-lip surfaces and 684 for oral cavity and its organs) using the publicly available anatomical data of a real man's cadaver produced by the Visible Human Project (National Institute of Health, USA) [7] and manual modelling in order to achieve maximum realism at the cost of increased modelling time. Especially for face modelling, we have used the CANDIDE-3 face model and its topology as a reference face for adapting it on the NIH data. Essentially, our work provides an extension of the CANDIDE3 towards the oral cavity which is important for medicaldental and speech pathology applications. In parallel to the work presented in this paper, the authors have developed a technique for adapting the model to any particular face and oral cavity of a human head by means of a Finite Elements Method (FEM). This technique will not be presented here due to lack of space. The final aim is to construct an animated, functional head model that will be used both in research and in medical education, as a virtual dental or medical patient. The next section describes our modelling approach step by step.

\section{ANATOMICAL BASED MODELLING}

\subsection{Data Preprocessing}

The Visible Human Project of the NIH produced two sets of cryosections (frozen traversal slices) taken from a male and a female cadaver which comprise the whole body. We have used the 377 images of these cryosections that correspond to the male's head. These images had a resolution of 2048 by 1216 pixels and 24-bit color depth. The resolution is 
considered adequate in order to easily identify all the internal tissues and conformations comprising the oral cavity of the cadaver therefore facilitating oral cavity modelling. Using the EIKONA 3D proprietary software package [8] we performed linear slice interpolation in $\mathrm{Z}$ axis by a factor of 3 in order to create a $3 \mathrm{D}$ image volume with cubic voxels. In order to concentrate in our area of interest we have cropped the volume so as to obtain roughly the front lower part of the head by just including the palatal bone. Using a mouse and a ray tracing-like, depth evaluation algorithm, we were able to obtain the 3D coordinates of any surface point on the rendered volume in order to construct the outer model surface, i.e. the face. Then, after having employed histogram equalization, we used the sectioning capabilities of the EIKONA 3D in order to identify the internal tissues and manually specify the nodes for the oral cavity surface of our model.

\subsection{Hierarchical-Scalable Model Structure}

Our 3D face and oral cavity model consists of two parts: the face model and the oral cavity model. Both of them have a hierarchical structure: they are comprised of elementary modules (e.g. right/left internal lower jaw, right/left lower teeth etc.) which can be combined at will to build more complex structures (e.g. lower jaw with teeth, upper jaw with hard palate etc.). Each module consists of nodes (point coordinates) and elements (triangles) data. Furthermore, the level of detail of our model (namely the number of nodes and elements) can be easily increased or decreased in order to model the 3D anatomical data at arbitrary levels of detail. This hierarchical and scalable approach provides us the flexibility to select an appropriate number of modules and/or nodes according to the targeted application.

\subsection{Face Modelling}

As mentioned earlier we have used CANDIDE-3 as our basic face wireframe within the hierarchical model. CANDIDE3 consists of 104 interconnected nodes forming 184 triangles and depicts a face in neutral posture and expression. Our initial purpose was to model only the external lower part of the male cadaver face using the same topology with the corresponding part of CANDIDE-3 in order to achieve compliance with MPEG-4 applications set to come (e.g. in medical tele-education). We have used only its lower 35 nodes and mapped them manually on corresponding facial points of the cadaver face chosen by inspection and anthropometric knowledge. These nodes cover two distinct regions of a human face namely the lips and the cheeks region. CANDIDE-3 is only the basic mode of our hierarchical face model. Extensions towards a much more detailed face model, e.g. the $8 K$ node model in [9], are possible.

\subsection{Mouth-Cheeks-Lips Modelling}

The hierarchy employed for mouth modelling creates the following anatomically distinct structures (modules): right and left internal lower jaw (87 nodes/112 triangles), right and left external lower jaw (87 nodes/112 triangles), right and left external upper jaw (93 nodes/120 triangles) and hard palate (63 nodes/92 triangles).

The basic structure in jaw modelling, either external or internal, was a 3 by 3 set of nodes covering the outer surface of each tooth's root (gum) either from outside (facing cheeks) or from inside (facing tongue) and continuing to genioglossus muscle in the later case. The upper edge of the set ( 3 nodes) covers the boundary between tooth enamel and soft tissue. The hard palate was modelled with a set of 31 perimetric nodes lying on the boundary between tooth enamel and soft tissue and 32 nodes scattered across palatal bone. In this phase the mouth model does not contain teeth. The internal cheeks and lips modelling, followed the one used for the cadaver's face by drawing perpendicular lines from the external feature nodes towards the mouth and by marking corresponding nodes in the intersection of these lines with the internal cheeks and lips surfaces. Special care has been taken in order to match the inner and outer (i.e. face) structures in the central lip area as to be able to animate both of them consistently. In order to estimate the accuracy of our models we mapped them on the cadaver's head volume as can be seen in Figures 1, 2. The model accuracy is very good for the mouth area but is not so good for the cheeks and lips, since the number of internal nodes had to match the corresponding external ones taken from CANDIDE-3.

\subsection{Teeth Modelling}

Although we have developed accurate models of teeth [10], we have resorted in a crude approximation of teeth as our basic model. Better teeth models can be used within the model hierarchy. We modelled the teeth according to the jaw hierarchy, separating the right and the left part of the lower-upper dental formations. For the first 4 frontal teeth of each dental arch (lower or upper) we used the 3 internal and 3 external boundary nodes of the respective jaw module plus 4 crest nodes for each tooth while for the rest we used 5 crest nodes in order to represent more accurately the larger mastication area. Thus we ended up with the following modules: left lower teeth (70 nodes/84 triangles), right lower teeth (75 nodes/94 triangles), left upper teeth (81 nodes/98 triangles), right upper teeth (75 nodes/94 triangles). The teeth are anchored on the jaw module, sharing the common boundary nodes and hence can be animated seamlessly with the jaws during speech articulation or mastication. The teeth integrated with the gums can be seen in Figure 3. 


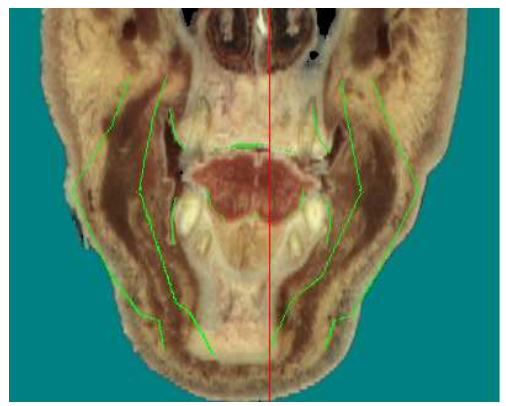

(a)

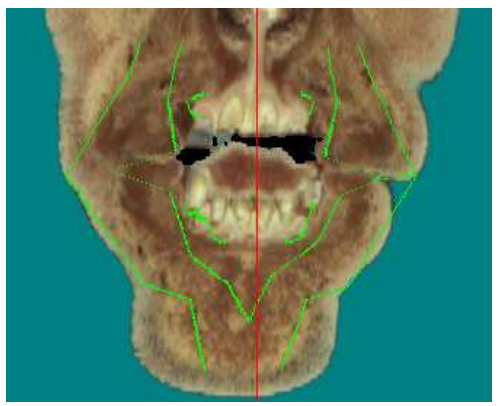

(b)

Fig. 1. Coronal view at plane 244 (a) and at plane 270 (b) for evaluating the accuracy of the model (green lines).

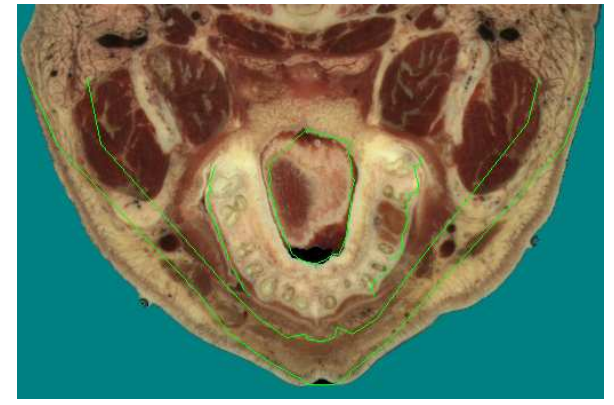

(a)

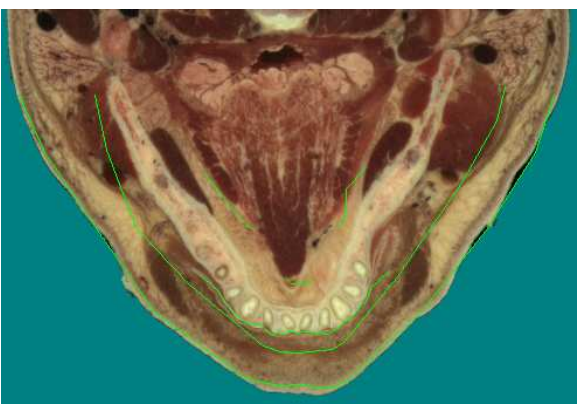

(b)

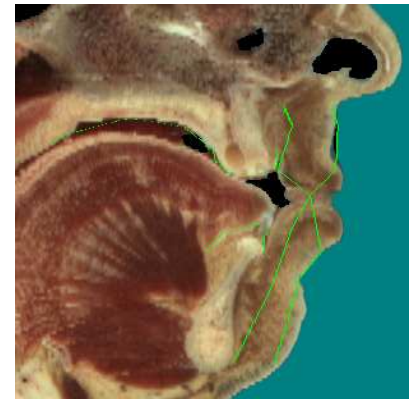

(c)

Fig. 2. Traversal view at plane 127 (a) and at plane 220 (b) for evaluating the accuracy of the model (green lines). (c) Sagittal view of the oral cavity model (green lines) superimposed on the anatomical data

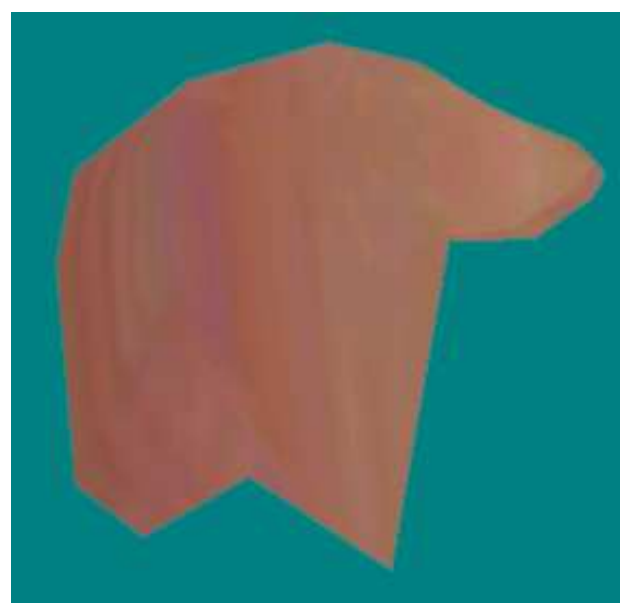

(a)

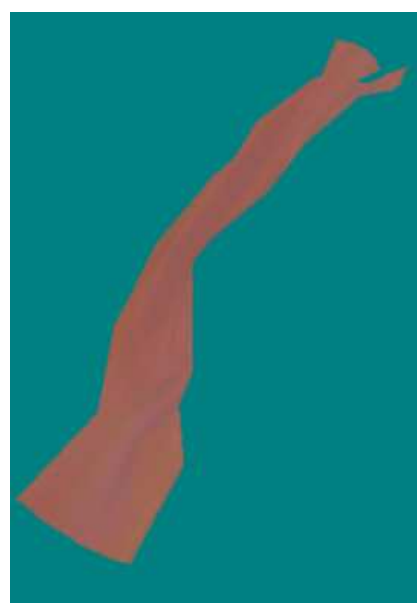

(b)

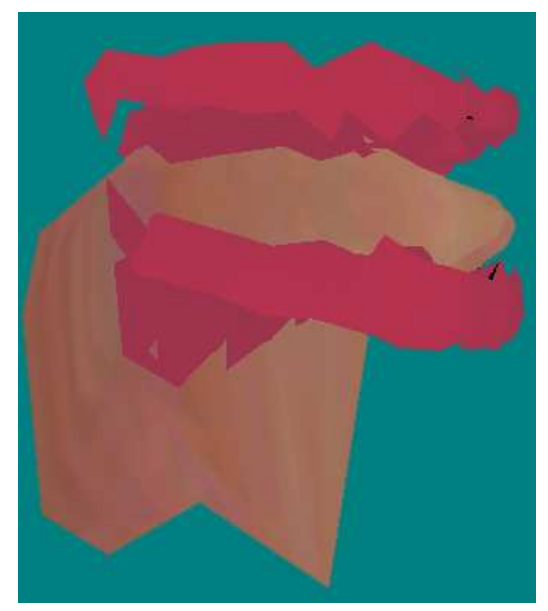

(c)

Fig. 4. (a) The tongue of the male head in "Visible Human Project" (front-left view), (b) The larynx and uvula side view of the male head in "V.H.P.", (c) The oral cavity of the male head in "V.H.P." (front-left view) 


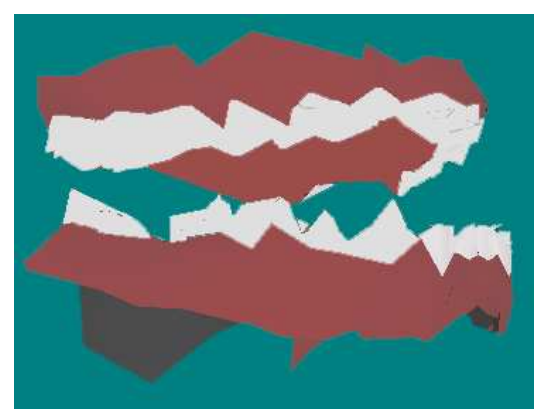

Fig. 3. Teeth model integrated with the gum model.

\subsection{Tongue-Larynx-Uvula Modelling}

For modelling tube-like structures like the tongue, the uvula and pharynx-larynx organs we have used tube cutting coronal or sagittal cross sections and taken nodes on the tube perimetry. For the tongue we have used 6 coronal planes with 8 perimetric nodes each, plus 1 coronal plane with 4 nodes, plus the tip of the tongue, forming a mesh with 53 nodes and 97 triangles. Similarly, for larynx modelling we have used 9 sagittal planes with 8 perimetric nodes each, forming a mesh of 72 nodes and 128 triangles. Here, we have omitted the extension of pharynx to oesophagus but included the uvula as a separate organ, since it plays a central role in oral cavity architecture as well as in speech production. The uvula model was created in analogy to the tongue mesh and consists of 25 nodes and 40 triangles. The final result is shown in Figure 4 for the tongue and the larynxuvula structures.

\subsection{Oral Cavity Synthesis}

Combining all the previously mentioned entities we have created a 550 nodes and 800 triangles mesh, which accurately depicts the cadaver's oral cavity as shown in Figure 4. This system is seamlessly combined with the CANDIDE-3 face model in order to be animated in the future, using extensions of the MPEG-4's "Facial Animation Object" which provides for real time animation of faces using Facial Definition Parameters (FDPs) and Facial Animation Parameters (FAPs) inherently present in CANDIDE-3. We do not overlook however the need for further extending the FDPs and FAPs in capturing the oral cavity's special geometry and animation peculiarities for speech-expression production.

\section{CONCLUSION}

In this paper we have presented a new model of the human face and oral cavity based on anatomical data of a real male cadaver (Visible Human Project). The derived model is modular, hierarchical and scalable while providing for
MPEG-4 compatibility in future animation attempts. Parallel to the static models we have developed a specialized mass-spring Finite Element technique (to be presented in a subsequent publication) as to be able to individualize our prototype models by means of a small driving set of characteristic points of the targeted surface. The final aim will be to construct functional virtual medical patients.

\section{ACKNOWLEDGEMENTS}

This work has been performed in the context of the SIMILAR Network of Excellence funded by the Commission of the European Communities.

\section{REFERENCES}

[1] F. Parke, "A parametric model for human faces, tech. report utec-csc-75-047," 1974.

[2] M. Rydfalk, "Candide, a parameterized face, report no. lith-isy-i-866," 1987.

[3] J. Ahlberg, "Candide-3 - an updated parameterized face, report no. lith-isy-r-2326," 2001.

[4] O. Engwall, "A 3d tongue model based on mri data," in Proc. of ICSLP2000, 2000, vol. III, pp. 901-904.

[5] Maureen Stone, Danielle Dick, Andrew Douglas, Edward Davis, and Cengizhan Ozturk, "5th seminar on speech production," 2000.

[6] Yves Laprie and Marie-Odile Berger, "Extraction of tongue contours in X-ray images with minimal user interaction," in ICSLP, CRIN-CNRS and INRIA-Lorraine BP 23954506 Vandoeuvre-1 Nancy FRANCE, 1996.

[7] Department of Health National Library of Medicine (USA) and National Institutes of Health Human Services, "Electronic imaging: Report of the board of regents," 1990.

[8] "Eikona software package, http://www.alphatecltd.com," .

[9] K. Kaehler, J. Haber, and H. Seidel, "Reanimating the dead: Reconstruction of expressive faces from skull data," in Proc., ACM SIGGRAPH, MPI Informatik, Saarbruecken, Germany, 2003, p. 5564.

[10] K. Lyroudia, G. Mikrogeorgis, P. Bakaloudi, E. Kechagias, N. Nikolaidis, and I. Pitas, "Virtual endodontics: three-dimensional teeth volume representations and their pulp cavity access," Journal of Endodontics, pp. 599-602, 2002. 